\section{OPEN JOURNAL SYSTEMS}

ISSN:2237-2202

\section{Available on line at Directory of Open Access Journals}

Journal of Hyperspectral Remote Sensing v.9, n.2 (2019) 80-87

www.periodicos.ufpe.br/revistas/jhrs

\section{Journal of Hyperspectral Remote Sensing www.ufpe.br/jhrs}

\title{
Interpolation methods applied to the population growth estimate
}

\author{
Aline M. Damasceno ; Paulo R. G. Sucupira Junior ${ }^{* *}$; Ana R. G. N. L. Salgueiro ${ }^{* * * *}$ Cynthia R. Duarte ${ }^{* * * *}$ \\ *Programa de Pós-Graduação em Geologia. Universidade Federal do Ceará - UFC. Fortaleza, CE, Brasil, E-mail: \\ alimoreiradamas@gmail.com.br \\ **Graduando em Estatística. Departamento de Estatística. Universidade Federal do Ceará - UFC. Fortaleza, CE, Brasil, E- \\ mail: paulogorayeb.statisticum@gmail.com.br \\ ****Programa de Pós-Graduação em Geologia. Universidade Federal do Ceará- UFC. Fortaleza, CE, Brazil, E-mail: \\ cynthia.duarte@ufc.br \\ ****Programa de Pós-Graduação em Geologia. Universidade Federal do Ceará- UFC. Fortaleza, CE, Brazil, E-mail: \\ geo.ritasalgueiro@gmail.com
}

Received 27 February 2019; accepted 15 May 2019

\begin{abstract}
Understanding the processes of variation of the population contingent of a given region is important and essential for related studies, mainly environmental planning issues. In this way, this article proposes to measure the population variation of a municipality, comparing two methods of population estimation, arithmetic and geometric, in order to choose the one that best quantified and represented the population changes of a region over the years. For this, census data from the years 1991,2000 and 2010 were used, which were also used to create a growth forecast for the year 2020, through the application of statistical estimation methods. In order to carry out the estimation, the numerical database of demographic censuses was used, thus adapting the results to the statistical functions. In this way, the study resulted in the identification of the method provided a better result in the implementation of the calculations. Thus, between the two methods used the geometric method, presented a better response, regarding the behavior and expression of the data for the case study analyzed.
\end{abstract}

Keyword: Methods of Estimation, Statistics, Population Dynamics.

\section{Métodos de interpolação aplicados à estimativa de crescimento da população}

\begin{abstract}
Resumo
Compreender os processos de variação do contingente populacional de uma determinada região, é algo importante e e imprescindível para estudos relacionados, principalmente a questões de planejamento ambiental. Desta forma, este artigo se propõe a mensurar da variação populacional de um Município, comparando dois métodos de estimação populacional, o aritmético e geométrico, com o objetivo de escolher aquele que melhor quantificou e representou as mudanças populacionais de uma região ao longo dos anos. Para isso, foram utilizados dados censitários dos anos de 1991, 2000 e 2010, que foram utilizados também para a criação de uma previsão de crescimento para o ano de 2020, através da aplicação de métodos estatísticos de estimação. Para a realização da estimação foram utilizados o banco de dados numéricos dos censos demográficos já realizados, adaptando assim os resultados as funções estatísticas. Desta forma, o estudo resultou na identificação do método proporcionou um melhor resultado diante da implementação dos cálculos. Sendo assim, dentre ambos os métodos utilizados o método geométrico, apresentou uma melhor resposta, com relação ao comportamento e expressão dos dados para o estudo de caso analisado.
\end{abstract}

Palavras-chave: métodos de estimação, estatística, dinâmica populacional.

\section{Introdução}

O município Cearense de São Gonçalo do Amarante possui um histórico envolvendo extensas ocupações e construções, desde melhorias na estrutura do município até grandes investimentos. $\mathrm{O}$ aumento de investimentos e a sua distribuição ao longo da zona costeira do município, ocorreu através do financiamento que favoreceu a realização e expansão das obras do CIPP (Complexo Industrial e Portuário do Pecém), com início das construções em
1995 e inauguração em 2002. Dessa maneira, pode-se considerar um marco para o início das diversas modificações na região (Magalhães, 2014).

Entende-se que o crescimento urbano quando feito de forma acelerada, principalmente em pequenos município e cidades médias tornam-se nocivos e consequentemente temática importante quando se discute questões relacionadas principalmente a qualidade de vida e qualidade ambiental. Vendrame (2000) salienta que quando o crescimento populacional ocorre de forma acelerada, 
traz como consequência avanços consideráveis da urbanização, que podem inclusive provocar impactos ambientais profundos, que podem até modificar as condições naturais de uma determinada região.

Aliado a esse crescimento populacional acelerado temos a falta de planejamento por parte das instituições políticas e governamentais, por justamente não esperar esse tipo de comportamento de uma certa população. Dessa maneira, podemos também associar à falta de planejamento de uma região o crescimento econômico, que permite a valorização dos espaços, permitindo o aumento das construções e modificações na região, o que acaba intensificando ainda mais a problemática da ocupação desordenada.

Além dos problemas estruturais, surgem problemas ambientais como a invasão de áreas de instabilidade ambiental, contribuindo para problema como: supressão dos componentes vegetacionais, ocupação de áreas vulneráveis como as dunas, e etc. Estes problemas quando agravados podem provocar assoreamento de rio e mananciais, surgimento de ilhas de calor, e etc, elevando assim a vulnerabilidade da região (Castro et al., 2012).

Devido a essas questões levantadas, a construção de estimativas populacionais realizadas em áreas com um crescimento populacional intenso, podem ser fortes ferramentas para auxiliar no planejamento e ordenamento territorial, pois muitas vezes o crescimento populacional, ainda não recebe o devido acompanhamento dos órgãos municipais, o que dificulta o acompanhamento e obtenção de dados para acompanhamento das transformações relacionada a esse tipo de estudo. A necessidade de coleta de informações relacionados as quantificações das ocupações populacionais e sobre a sua dinâmica ao longo dos anos, são importantes para a criação dos prognósticos, para que os mesmos possam vim a suprir de forma rápida e eficaz as demandas da população, da cidade e do meio ambiente (Iwai, 2003).

Considerando assim as questões expostas, a realização deste estudo foi voltada para compreender a dinâmica populacional, e para isso foram utilizados métodos de interpolação entre pontos conhecidos, pois estes tipos de métodos permitem a avaliação a partir de pontos conhecidos, para assim realizar estimações. Levando em consideração que o objetivo deste estudo é identificar o aumento populacional no município de São Gonçalo do Amarante, utilizou-se como pontos conhecidos as populações recenseadas nos períodos censitários realizados pelo Instituto Brasileiro de Geografia e Estatística. Portanto, a utilização dos censos em estudos dessa natureza, se tornam essenciais quando se deseja estimar a população de uma cidade, estado ou região em um período intercensitário além de compor um banco de dados que podem ser acessados constantemente (Godoy, 2012).

Nesse sentido, o trabalho possui como motivação principal, modelar o crescimento populacional dos distritos que constituem a região do município de São Gonçalo do Amarante- CE, através da aplicação de métodos de interpolação, utilizando especificamente os métodos aritméticos e geométricos.

\section{Material e métodos}

O município de São Gonçalo do Amarante, pertence à Região Metropolitana de Fortaleza e dista $60 \mathrm{Km}$ da capital Cearense. São Gonçalo do Amarante possui em seus limites os municípios de Caucaia, Pentecoste, São Luiz do Curu, Trairi, Paraipaba e Paracuru (Figura 1). As vias de acesso são através das Rodovias BR-222 e CE-423 ou também pela Rodovia CE-085, chamada de Rodovia do Sol Poente ou popularmente como Via Estruturante.

Para a realização da pesquisa, foram utilizados mapas de uso do solo para cada ano levando em consideração a sua correspondência ao tempo delimitado para os censos demográficos realizados.

Os distritos utilizados para o estudo são os que integram essa região atualmente, sendo um total de oito sendo eles: Croatá, Pécem, São Gonçalo do Amarante, Serrote, Síupe, Taíba, Umarituba e Cágado. Cada distrito foi avaliado a partir de sua ocupação ao longo dos anos, de acordo com as imagens de satélite coletadas para cada ano especifico dos censos realizados e os dados de população residente coletada pelos censos demográficos, que são a fonte de dados mais utilizada para o levantamento da população, pois, são as mais completas em termos de cobertura do território. Com isso foi possível quantificar e identificar as mudanças ocorridas através dos métodos interpoladores aplicados, para pôr fim selecionar e identificar aquele que obteve a melhor representação e resposta ao objetivo do estudo, que é quantificar o crescimento populacional da área.

Os mapas foram construídos a partir de imagens LandsatTM 5, que possuem uma resolução de 30 metros, e que são importantes quando se espera uma maior captação da área de imageamento, além disso, estas imagens possuem uma temporalidade espacial no qual permite o estudo multitemporal, permitindo acompanhar as mudanças no espaço de forma temporal. Para a seleção das imagens o critério utilizado foram imagens que apresentassem pouca cobertura de nuvens, feito isso os anos selecionados foram 1991, 1999, 2007 e 2018. Posteriormente foram realizados o georreferenciamento e correções 
geométricas das imagens para a confecção dos mapas de uso do solo. Por fim, iniciou-se a aplicação dos modelos matemáticos para a construção do prognóstico populacional. As projeções foram realizadas a partir de dois métodos matemáticos, os métodos geométricos e aritméticos, a fim de identificar e determinar as tendências de crescimento populacional na região em função do melhor resultado determinado pela resposta dos dados interpolados e através da variação da modelagem matemática.

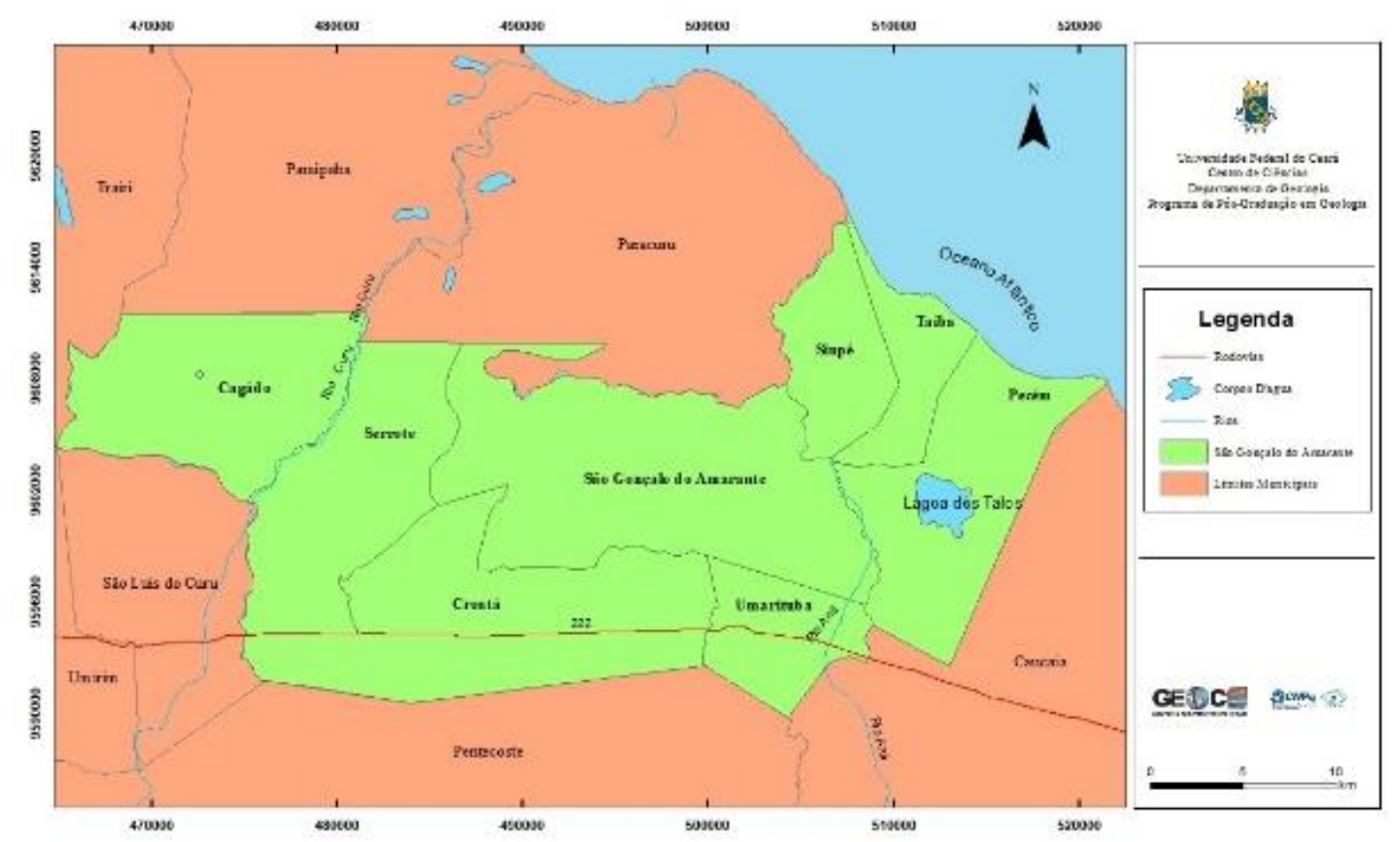

Figura 1 - Mapa de Localização do município de São Gonçalo do Amarante-CE. Fonte: Própria Autora .

\section{Interpolação populacional}

$\mathrm{O}$ conceito de interpolação foi definido em 1980 por Shryock e Siegel (2004). De acordo com os pesquisadores, o conceito de interpolação remete a possibilidade de inferir valores intermediários tendo como base uma série de dados conhecidos, para assim determinar sua representação matemática e gráfica. Segundo Cerqueira e Givisiez (2004), a aplicação destas técnicas permite a aproximação de dados de população em datas não cobertas por censos, através da estimativa em um tempo $(t)$ com base numa taxa de crescimento $(r)$, possibilitando assim uma projeção de dados populacionais com uma grande precisão matemática permitindo assim uma maior confiabilidade dos resultados, pois apesar dos erros associados as estimativas são confiáveis. Dessa forma, os métodos matemáticos interpoladores mais utilizados para o cálculo da estimativa populacional são os geométricos e os aritmético. Sendo estes os escolhidos para a realização deste trabalho.

\section{Metodo geométrico}

Este método analisa o crescimento populacional de uma população levando em consideração uma fase de crescimento acelerado, no qual o mesmo acompanha a forma de uma curva exponencial. As estimativas de crescimento da população a partir do uso deste método, são obtidas de acordo com a formula abaixo. No qual a taxa de crescimento ( $\mathrm{q}$ ) é subtraída do quociente entre a população final $(\mathrm{Pf})$ e a população no começo do período da análise, considerado a (Po).

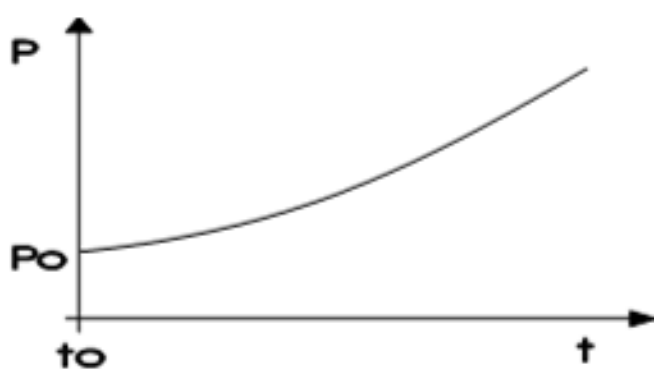

Onde:

$\mathrm{q}=$ Taxa de crescimento geométrico;

$\mathrm{P}_{\mathrm{O}}=$ População inicial do último censo conhecido;

to $=$ Ano do último censo ;

Pf = População final;

tf = Ano necessário (início, meio e fim da ).

Dessa maneira as taxas futuras de crescimento geométrico são usadas a partir das datas 
passadas como exemplificado abaixo, a partir dos dados dos censos:

$\mathrm{q} 1=(\mathrm{P} 1991 / 2000)^{\wedge} 1 /(1991-2000)$

$\mathrm{q} 2=(\mathrm{P} 2000 / \mathrm{P} 1991)^{\wedge} 1 /(2000-1991)$

$\mathrm{q} 3=(\mathrm{P} 2010 / \mathrm{P} 2000)^{\wedge} 1 /(2010-2000)$

\section{Método aritmético}

$\mathrm{O}$ método aritmético pressupõe uma taxa de crescimento constante para os anos que seguem a partir de dados conhecidos, por exemplo, a população do último censo Tsutiya (2006). Este método pressupõe o crescimento de uma população sob a forma de uma projeção aritmética, ou seja, sua amostragem é bastante semelhante a uma reta. Quando relacionado a população, entende-se que o crescimento da população se mantem constante pelo ano, evoluindo segundo a linha reta, que representa seu resultado.

A fórmula que representa esse método é dada pela expressão:

$$
\begin{aligned}
& P_{x}=P_{u}+(x-u) r \\
& r=\frac{P_{u}-P_{p}}{u-p}
\end{aligned}
$$

Onde:

$\mathrm{r}=$ taxa de crescimento anual;

$\mathrm{Pu}=$ população do último censo;

$\mathrm{Pp}=$ população do penúltimo censo;

$\mathrm{p}=$ data do penúltimo censo; $\mathrm{e}$

$\mathrm{x}=$ data que se quer estimar a população

\section{Resultados e discussão}

Estudos das projeções demográficas que utilizam métodos estatísticos e matemáticos são utilizados com frequência devido a constante mobilidade populacional, sendo necessário assim estudos que permitam delimitar planos de estratégia levando em consideração as diferentes características demográficas. As compreensões dessas características ajudam na percepção dos impactos sociais referentes a características que podem ser analisadas posteriormente como mudanças no nível de coesão, vitalidade, confiança e demografia de um grupo populacional.

Estudos de estimação populacional possuem dessa forma o objetivo de utilizar dados populacionais passados, utilizando, series históricas de uma variável, relacionado ao crescimento populacional. (Santos, 1989; Waldvogel, 1998; Jardim, 2001). A escolha do método a ser utilizada é interessante para definir o tipo de estudo no qual se aplicará esses dados de series históricas, podendo ser do tipo estimação ou projeção. A exemplo de estimações, temos estudos utilizando imagens de satélites, ou fotografias aéreas, realizadas na Austrália por Bell (1997). No Brasil, Saboya (2000) e Kempel (2003) também trouxeram contribuições ao uso de métodos analíticos associados a imagens de satélite para a detecção de expansões urbanas.

Com relação à projeção de populações, utilizando setores censitários, Yi (1991), possui uma proposta mais semelhante ao uso de métodos de interpolação e estimativas de projeção para domicilio, para isso o autor propõe a utilização de censos demográfico de 1991 e 2000 . No entanto, diferente da proposto deste trabalho, esses estudos aplicam métodos mais robustos e estatisticamente mais trabalhados.

No caso desta análise de estimação, o primeiro aspecto relevante é em relação a constituição espacial do município de São Gonçalo do Amarante. O município passou por mudanças ao longo de seu desenvolvimento, a área que corresponde hoje aos oito distritos, antes era ocupada apenas por sete distritos. Eram eles: Croata, Pecém, São Gonçalo do Amarante, Serrote, Síupe, Taiba e Umarituba. No ano de 2006 ocorre a criação do oitavo distrito, Cágado, constituído de parte o Distrito de Serrote de acordo com a Lei do município, datada de 12 de dezembro de 2006. Essa mudança ocorrida no município trouxe algumas ressalvas quanto as aplicações dos métodos de estimativa populacional para o Distrito de Cagado, pois como o método utiliza a relação da avaliação da população residente no município ao longo dos anos de acordo com os censos de 1991, 2000 e 2010, não possível a coleta de dados referentes aos anos inferiores a 2010, o que ocasionou a ausência de informações para as estimativas, especificamente para este distrito. (Tabela 1). No entanto, com relação aos demais Distritos, alguns vinham apresentando um crescimento considerável, que foi possível observar em todos desde de 1991. No ano de 2000 o distrito de Serrote, após o processo de constituição do distrito de Cagado, apresentou uma diminuição de sua população, que também vinha em ascendência. Com a inauguração do Complexo Portuário do Pecém em 2005, o ano de 2010 apresenta valores significativos com relação a população, principalmente nos distritos influência direta do Complexo Portuário do Pecém, dentre eles com destaque para o distrito do Pecém e são Gonçalo do Amarante. 
Tabela 1 - Estimativas Populacional para os Métodos Geométricos e Aritméticas.

\begin{tabular}{cccccc}
\hline Municípios & $\mathbf{1 9 9 1}$ & $\mathbf{2 0 0 0}$ & $\mathbf{2 0 1 0}$ & $\mathbf{2 0 1 8}(\mathbf{A})$ & $\mathbf{2 0 1 8}(\mathbf{B})$ \\
\hline Croata & 5.049 & 5.638 & 6.400 & 7.010 & 7.083 \\
Pecém & 5.362 & 7.460 & 9.156 & 10.513 & 10.789 \\
São Gonçalo do amarante & 6.525 & 7.535 & 11.212 & 14.154 & 15.409 \\
Serrote & 5.890 & 6.880 & 2.991 & -120 & 1.536 \\
Siupé & 2.413 & 2.942 & 3.658 & 4.231 & 4.354 \\
Taiba & 2.939 & 3.911 & 5.104 & 6.058 & 6.316 \\
Umarituba & 1.108 & 1.242 & 1.428 & 1.577 & 6.567 \\
Cagado & $\mathrm{x}$ & $\mathrm{x}$ & 3.941 & $\mathrm{x}$ & $\mathrm{x}$ \\
\hline
\end{tabular}

Analisando os resultados referentes ao método Aritmético e Geométrico, percebe-se um valor negativo associado ao município de Serrote, quando modelado pela função aritmética, no entanto para o mesmo distrito, quando modelado pelo Interpolador geométrico, nota-se uma variação positiva. Com relação a modelagem negativa do interpolador aritmético, acredita-se que essa modificação possa ter ocorrido devido a modificação brusca de valores populacionais inseridos no cálculo. No ano de 1991 a 2000 é possível observar um padrão de crescimento positivo para a região, mas que em 2010 tem uma diminuição considerável. A esta mudança nos valores, associa-se a criação do novo Distrito em 2006, o Cagádo, que também apresentou problemas em sua estimação, como citado anteriormente. A explicação para essas variações negativas que ocorrerem com o Distrito de Serrote, (Figura 2), está associada justamente a essa mudança na sequência de algoritmos utilizados antes e depois para a estimação do valor para 2018 e para os demais anos, devido a situação apresentar $\mathrm{r}<0$, o resultado gerado seguiu a tendência de uma condição estacionaria, decrescente ou constante, características de estimativas Aritméticas (Maia, 2011).

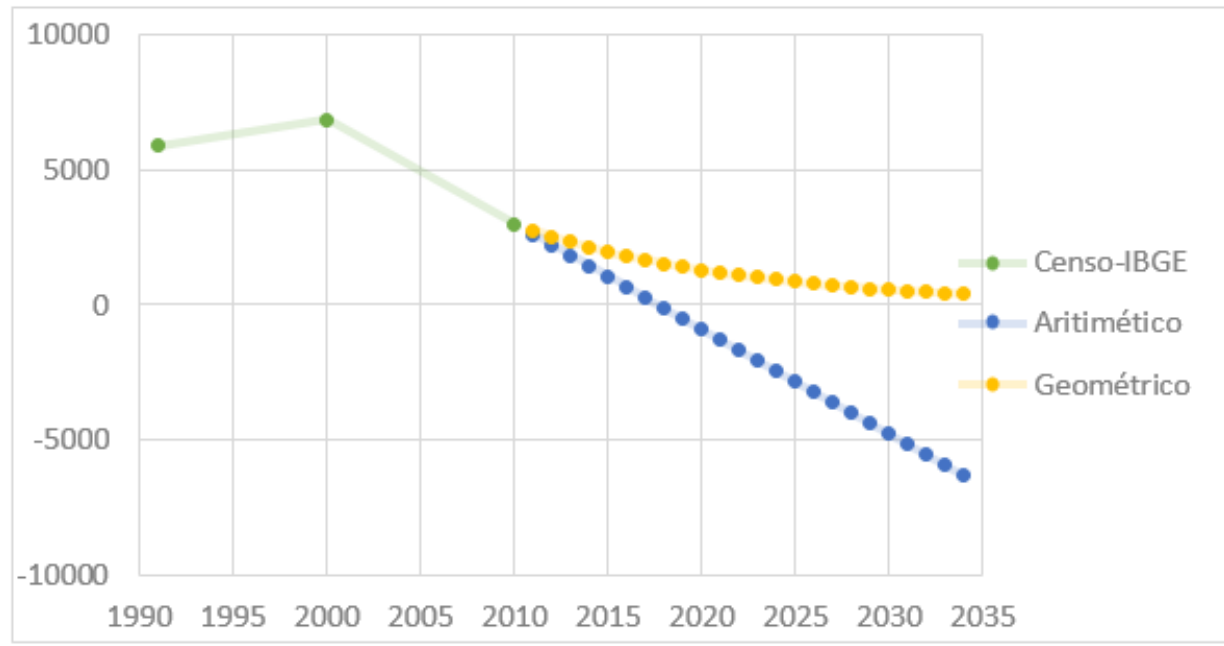

Figura 2 - Estimativas crescimento populacional feitas para o Distrito de Serrote-CE.

Já o método geométrico, (Figura 3), se mostrou eficiente ao se comportar de forma positiva e linear em relação a variação da população para os demais Distritos avaliados. Aplicando o mesmo método com dados de setores censitários, obtivemos apenas o déficit de dados anteriores para o Distrito de Cagado, pois por ter sido criado em 2006 apenas começou a ser recenseado oficialmente no ano de 2010, não foi possível estimar as informações para o mesmo. No entanto, para os demais distritos foi possível relacionar de forma satisfatória as variações com relação ao crescimento populacional e ao método de estimação, pois não apresentaram valores decrescentes e fora o padrão de resposta de uma progressão geométrica, que seria a associação do fator anterior para identificar $\mathrm{o}$ fator seguinte, mantendo-se assim um valor proporcional. Esse método foi o que mais possibilitou acompanhar o 
desenvolvimento temporal continuo do crescimento populacional para o município de São Gonçalo do Amarante- CE.

Com relação ao crescimento identificado pelo método Geométrico acredita-se que a expansão realizada no Complexo Portuário do Pecém, que segundo Alcântara e Avelino (2013), em 1985, o Governo Federal, através da Petrobras, determinou a construção de uma nova refinaria no Nordeste do Brasil. Essa construção entra em vigor, após dez anos de disputa, e em 1995, o Ceará ganha uma refinaria. Por razões econômico-financeiras, este empreendimento não vai adiante, dando lugar à construção do Porto de Pecém.

O início das operações comerciais deste novo porto em novembro de 2001 e sua posterior inauguração em março de 2002, no entanto no de 1995 a 1999, a região do distrito do Pecém teve sua primeira onda das desapropriações, esse processo, teve como saldo centenas de famílias expulsas da terra, sendo algumas alojadas nos assentamentos em outros municípios cearenses (Nóbrega e Martins, 2010). Esses movimentos de migração da população do município podem ser relacionados ao aumento populacional observado, nos demais distritos ao longo dos anos de 2000.

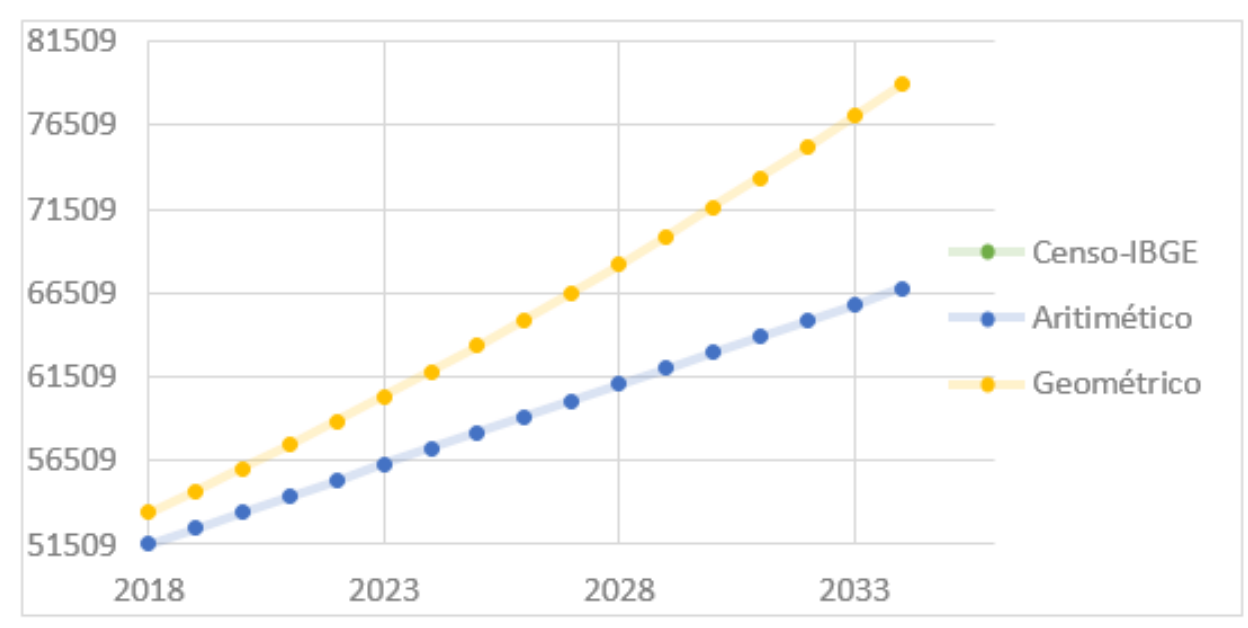

Figura 3 - Projeção Populacional para o município de São Gonçalo do Amarante-CE.

No ano de 2002, ocorre a instalação definitiva do CIPP (CIPP, 2015). Segundo Soja (1993) nesse período de adaptação do Porto, aconteceu uma espécie de revolução demográfica, afastando centenas de pessoas de seus habitats naturais, lançando-as em outra direção para novas vidas, em mobilidade do trabalho. Foi nesse momento de crescimento urbano rápido que emergiram vários dos problemas urbanos. De acordo com Magalhães (2014) a população do município de São Gonçalo do Amarante passa a ter um aumento considerável com relação ao contingente populacional de 2010, sendo observado que a população anteriormente caracterizada como sendo predominantemente rural passa a se tornar mais urbana. A essas mudanças ainda é possível correlacionar a data de anexação do distrito de Cágado ao Distrito de Serrote, que ocorreu em 2006, o que ocasionou também uma diminuição considerável do contingente populacional do Distrito Serrote também ocorrida entre os anos 2000 e 2010, demostrando a presença constante de movimentos de dispersão populacional.

De acordo com o banco de dados de indicadores socioeconômicos do Instituto de Pesquisa e Estratégia Econômica do Ceará (IPECE, 2000), em 2000 a população era estimada em 7.460 habitantes, enquanto que em 2010 subiu para 9.156 habitantes. Este tem ocorrido como um resultado do crescimento econômico, visto que o número de empregos formais, de acordo com o mesmo Instituto, no ano de $2007 \mathrm{em}$ São Gonçalo do Amarante era de 3.675 comparado aos empregos gerados em 2010, que foi de 7.821.

Analisando a proporção de crescimento, a Tabela 2 mostras do resultado obtido pelo método Aritmético e Geométrico, para as populações futuras, no município de são Gonçalo do Amarante. 
Tabela 2 - Dados de estimação populacional para o município de São Gonçalo do Amarante-CE.

\begin{tabular}{ccc}
\hline Ano & $\begin{array}{c}\text { População total pelo } \\
\text { método Aritmético }\end{array}$ & $\begin{array}{c}\text { População total pelo } \\
\text { método Geométrico }\end{array}$ \\
\hline 2018 & 51509 & 53377 \\
2019 & 52462 & 54699 \\
2020 & 534145 & 56053 \\
2021 & 54366 & 57442 \\
2022 & 55319 & 58864 \\
2023 & 56271 & 60322 \\
2024 & 57224 & 61815 \\
2025 & 58176 & 63346 \\
2026 & 59128 & 64915 \\
2027 & 60081 & 66522 \\
2028 & 61033 & 68170 \\
2029 & 61986 & 69858 \\
2030 & 62938 & 71588 \\
2031 & 63890 & 73361 \\
2032 & 64843 & 75177 \\
2033 & 65795 & 77039 \\
2034 & 66748 & 78947 \\
\hline
\end{tabular}

\section{Conclusões}

Este trabalho exemplifica dois tipos de métodos de estimação populacional. Quando estes métodos são analisados, é possível interpretar a necessidade da utilização e do aperfeiçoamento dos métodos fim de melhorar de forma matemática, a estimativa de erros associados a essa estimação.

No entanto, com os métodos aplicados, ainda sim foram possíveis se obter uma visão global e direcionada para a compreensão do processo temporal de crescimento populacional, assim como estimar o comportamento populacional futuro.

Vale ressaltar também que a partir dos resultados encontrados, foi possível identificar pontos ao longo do processo de ocupação do município que que necessitam de uma intervenção estratégica com relação aos planejamentos das cidades, e principalmente ao meio ambiente. Por fim, com relação aos métodos aplicados para a estimação do crescimento populacional enfatiza-se os problemas encontrados com relação a falta de informações referente a criação do novo Distrito, o Cagado, o que ocasionou problemas nas estimações realizadas pelo método Aritmético. O resultado gerado através do método Geométrico, juntamente com as estimativas encontradas se mostraram satisfatórias, visto que foi possível entender e interpretar o funcionamento da ocupação do município, permitindo também, traçar um perfil de crescimento, analisado conjunturalmente com as mudanças ocorridas ao longo do tempo e espaço no município de São Gonçalo do Amarante. Por fim, recomenda-se a criação de um extenso banco de dados adicionados a dados de uso do solo, para que juntamente com estimativas realizadas e os diagnósticos encontrados, estabelecer um monitoramento constante das modificações populacionais afim de utilizar esse banco de dados como subsidio para aplicação de uma nova análise comparativa para o censo de 2020.

\section{Agradecimentos}

Os autores agradecem a CAPES pelo fornecimento da bolsa de pós-graduação permitindo assim a realização desta pesquisa.

\section{Referências}

Alcântara, S., Avelino, F.R.F., 2013. Transformações Socioambientais: uma visão sobre o desenvolvimento no Pecém. Revista Gestão \& Desenvolvimento 1, 1-18.

Bell, M. et al., 1997. Forecasting population growth and housing development for small areas using GIS. Conferência mundial de população, Pequim.

Castro, A.S.F., Moro, M.F., Menezes, M.O.T., 2012. O Complexo Vegetacional da Zona Litorânea no Ceará: Pecém, São Gonçalo do Amarante. Acta Botanica Brasilica 26, 108-124.

Cerqueira, C.A., Givisiez, G.H.N., 2004. Conceitos básicos em demografia e dinâmica demográfica brasileira, In: Rios-Neto, E.L.G., Riani, J.deL. (Org.). Introdução à Demografia da Educação. Ed. ABEP, Campinas.

CIPP. Complexo Industrial e Portuário do Pecém, 2015. 
http://www.adece.ce.gov.br/index.php/porto-dopecem.

Godoy, W.A.C., 2012. Padrões ecológicos e tamanhos populacionais críticos: sensores importantes em modelagem e controle. Oecologia Australis 16, p. 32-42.

IPECE. Instituto de Pesquisa e Estratégia Econômica do Ceará, 2000. Informações Básicas Municipais. IPECE, Fortaleza.

Iwai, O.K., 2003. Mapeamento do uso do solo urbano no município de São José dos Campos, através de imagens de satélite. Dissertação (Mestrado). São Paulo, Escola Politécnica da USP.

Jardim, M.L.T., 2001. Uso de variables sintomáticas para estimar la distribución espacial de la población. Notas de Población 71, 21-49.

Kempel., S., 2003. Geoinformação para estudos demográficos: representação espacial de dados de população para Amazônia. Tese (Doutorado). São Paulo, Escola Politécnica da USP.

Magalhães, C., 2014. Alterações socioespaciais na planície litorânea de São Gonçalo do Amarante CE. Dissertação (Mestrado). Fortaleza, UFC.

Maia, J.D., 2011. Progressões aritméticas e geométricas. Trabalho de Conclusão de Curso (Graduação). Campina Grande, UEPB.

Nóbrega, L.N., Martins, M.P.M.J., 2010. O povo indígena Anacé e o Complexo Industrial e Portuário do Pecém: tessituras socioambientais de um Admirável Mundo Novo. Dissertação (Mestrado). Fortaleza, UFC.

Saboya, R.T., 2000. Análises espaciais em planejamento urbano. Revista Brasileira de Estudos Urbanos e Regionais 3, 61-79.

Santos, T.F., 1989. Projeções de população de Pernambuco, desagregada por microrregiões, até o ano de 2010: aplicação de métodos alternativos. Dissertação (Mestrado). Belo Horizonte, UFMG.

Shryock, H.S.E., Siegel., S.J., 2004. The Methods and Material of Demography. U. S. Government printing office, Washington.

Soja, E.W., 1993. Geografias pós-modernas: a reafirmação do espaço na teoria critica. Tradução de Vera Ribeiro. Zahar, Rio de Janeiro.

Tsutiya, M., 2006. Abastecimento de Água. 3. ed. Escola Politécnica da USP, São Paulo.

Vendrame, I.F., 2000. Análise do crescimento urbano e seus efeitos na mudança da dinâmica de escoamento superficial da bacia do Pararangaba. INPE, São José dos Campos.

Waldvogel, B.C., 1998. Técnicas de Projeção Populacional para o Planejamento Regional. CEDEPLAR, Belo horizonte. 